\title{
Harnessing cell-material interaction to control cell fate: design principle of advanced functional hydrogel materials ${ }^{\dagger}$
}

\author{
RAJAT K DAS* \\ Materials Science Centre, Indian Institute of Technology, Kharagpur, West Bengal, India \\ E-mail: rajat@matsc.iitkgp.ernet.in
}

MS received 15 August 2017; revised 29 September 2017; accepted 4 October 2017; published online 26 October 2017

\begin{abstract}
Physical (topography, stiffness) and chemical instructions encoded in the extracellular environment govern cell behaviour. Understanding these interactions as they operate in native extracellular matrix and capturing the complexity of the spatiotemporal presentation of multiple such signals in synthetic scaffolds is a key to develop advanced functional materials for tissue engineering applications. This review discusses the advancement in engineered hydrogel materials as such scaffold to control cell fate.
\end{abstract}

Keywords. Extracellular matrix; hydrogel; biomaterials; tissue engineering.

\section{Introduction}

Research towards the development of bio-inspired synthetic extracellular matrix (ECM) mimetic materials is advancing at a rapid pace. Functional biomaterials are expected to play a pivotal role in the field of tissue engineering and regenerative medicine especially at the level of complexity composed from the interplay of chemical triggers with material properties such as matrix (scaffold) stiffness and topography. The extracellular matrix (ECM) is composed of a mixture of insoluble hydrated fibrillar polymers such as fibrinogen and collagen. ECM stiffness and topography provide the physical cues; soluble macromolecules (chemokines) in combination with larger molecules embedded on the surfaces of neighbouring cells (proteins and ligands) present chemical signals. The ultimate fate of a cell (differentiate, proliferate, migrate, apoptose or perform other specific functions) is a result of its response to the complex spatio-temporal biochemical and biomechanical cues from the extracellular microenvironment.

Hydrogels, because of their biocompatibility, water retention capacity and the ability to transport oxygen and nutrients, are promising candidates as scaffolds for cells, not only for fundamental studies elucidating cellmaterial interaction, but also for research towards eventual practical applications in tissue engineering. ${ }^{1-3}$ This

\footnotetext{
*For correspondence

${ }^{\dagger}$ Dedicated to Professor Uday Maitra on the occasion of his $60^{\text {th }}$ Birth Anniversary.
}

critical review will discuss (from a material chemist's perspective), in the context of state of the art development of hydrogel materials for tissue engineering applications, integration of key ECM parameters into programmed semi-synthetic and synthetic extracellular matrix materials in comparison to naturally available materials.

\section{Cell-matrix interaction: biological triggers on cellular fate}

All the important cellular events, for example, adhesion, spreading, proliferation and differentiation, are governed by the interactions of the cells with their surrounding matrix. In this section, we shall discuss the present understanding of these events from an overall general perspective, to lay the basis for a detailed discussion (in the following sections) of the specific matrix properties that underpin these interactions, encompassing both mechanical and chemical signals from the matrix, and their ramifications in the design principles of next generation biomaterials.

Cell surface adhesion receptors integrins play an important role as anchors and are involved in important cellular processes such as embryogenesis, differentiation, immune responses, wound healing and hemostasis. Integrins consist of two supramolecular transmembrane subunits termed $\alpha$ and $\beta$. In the process of integrin mediated cellular adhesion, a cascade of different events occurs: attachment, spreading, organization of actin cytoskeleton and the formation of focal adhesions. In the initial stages of cell-matrix interaction, focal adhesion (FA) complex formation occurs between the integrins and the extracellular matrix ligands [for example, RGD (arginine-glycine-aspartate amino acid sequence), a 
minimal adhesive sequence identified in fibronectin, an ECM component]. It is through this complex that the mechanical information of the matrix is transmitted to the cytoskeletal actin. Concurrently, chemical signals are also received through the focal adhesions (for example, in the case of growth factor receptor-growth factor binding). This event is basically the equivalent of the cells 'sensing' their surrounding microenvironment. Several cytoskeletal proteins, such as, talin, filamin, ILK, etc. that interact with integrin $\alpha \beta$ cytodomain, are believed to be recruited in the FA complex. Although the mechanisms of the force transmission pathway are still not well understood, talin binding to the integrin $\beta$ cytoplasmic domain is believed to be the crucial step for the activation of integrins and to establish ECM-integrin-talinactin link in the early stages of FA complex formation. ${ }^{4-6}$ A key role for another protein, vinculin, in the stabilization of the FA complex and the effective force transmission from the matrix to the cytoskeletal actin, has been established. The globular head of talin connects to integrin in the FA complex and the rod-like tail is attached to actin in cytoskeleton. The transmission of signal (both mechanical as well as chemical) through the FA complex into the cytoskeleton initiates a series of events and controls specific gene expression in the nucleus (for example, Rho Kinase, ROCK; Focal adhesion kinase (FAK) pathways are activated in the cytoskeleton; phosphorylation and nuclear translocation of Smad proteins occur in the case of osteogenic differentiation). Phosphorylation of non-muscle myosin II, a cytoskeletal motor, leads to actin bundle formation and stress actin fiber generation. It has been speculated that this actomyosin contraction event unfolds the talin rod and exposes the buried binding sites for vinculin, which is then recruited into the FA complex through talin-vinculin interaction. ${ }^{5} \mathrm{~A}$ feedback mechanism operates in executing the whole process of matrix mechanics 'sensing' by the cells. Thus, when the focal adhesions develop, the cells pull on the matrix by force generated through actomyosin contraction. If the matrix is stiff, and is thus able to resist this deformation at the adhesion sites, then the focal adhesions mature (clustering of integrins) and the cells subsequently pull on the matrix with greater force. It thus becomes clear from the discussions in this section that the cell-matrix interaction is a complex phenomenon in the physiological context. The mechanical and chemical signals from the matrix operate in tandem and determine the fate of the stem cells (cytoskeletal actin re-organization, gene expression, etc.).

In attempting to decode different facets of these interactions, studies have focused on synthetic and biogenic extracellular matrix mimetic substrates programmed to present specific mechanical/chemical signals to elicit specific cell responses (Figure 1).

\section{Natural ECM materials for cell culture}

Hydrogels based on natural materials such as, fibrin, collagen, HA, etc., benefit from the fact that they already contain cell interactive sites. Natural ECM materials have played an important role in tissue engineering applications. These materials have been successfully used as scaffolds to host transplanted cells from donor, and then implanted as grafts to repair tissue defects in the donor. For example, collagen scaffold seeded with fibroblast cells from rat donor was shown to contract and generate a dermal substrate on which differentiation of epidermal cells was possible leading to epidarmis keratinization in vitro. This live tissue was successfully implanted in the skin defect of a rat model. ${ }^{7}$ Collagenglycosaminoglycan copolymers have been reported to induce skin morphogenesis. ${ }^{8}$ Besides, scaffolds from natural materials also support cell infiltration, important for formation of vascular network in the grafted tissue, its remodelling and subsequent integration. As an example, a collagen/chitosan porous scaffold (cross-linked with glutaraldehyde to improve mechanical strength) has been shown to support fibroblast infiltration from surrounding tissues, when implanted in rabbit ear. ${ }^{9}$ Most importantly, natural ECM based scaffolds have provided useful model systems to identify principles that would help design synthetic biomaterials with engineered properties. The following example will illustrate this point: the well known affinity of transforming growth factor $\beta$ (TGF- $\beta$ ) (an important growth factor for bone repair) towards heparin was exploited to design heparin conjugated collagen scaffolds as materials for bone regeneration, wherein the heparin sites provided an anchoring point for the growth factor, which could then act as a controlled release system of the growth factor to the cells. ${ }^{10}$ This principle has been integrated into the design of bioinspired synthetic scaffolds. ${ }^{11}$ This issue will be discussed in more detail in a later section where the presentation of growth factors in engineered hydrogels will be covered.

Despite several useful applications as detailed above, ECM derived materials require difficult purification procedures and carry the risks of immunogenicity and disease transmission. Batch to batch variation (after isolation from biological tissue) further limits their applications.

\section{ECM mimetic hydrogels: design principles}

This section discusses the key design parameters that need to be incorporated into the programmed ECM mimetic hydrogel materials (synthetic and semisynthetic).

\subsection{Biodegradability}

The matrix needs to be biodegradable. The cells need to be able to remodel the matrix network in order 


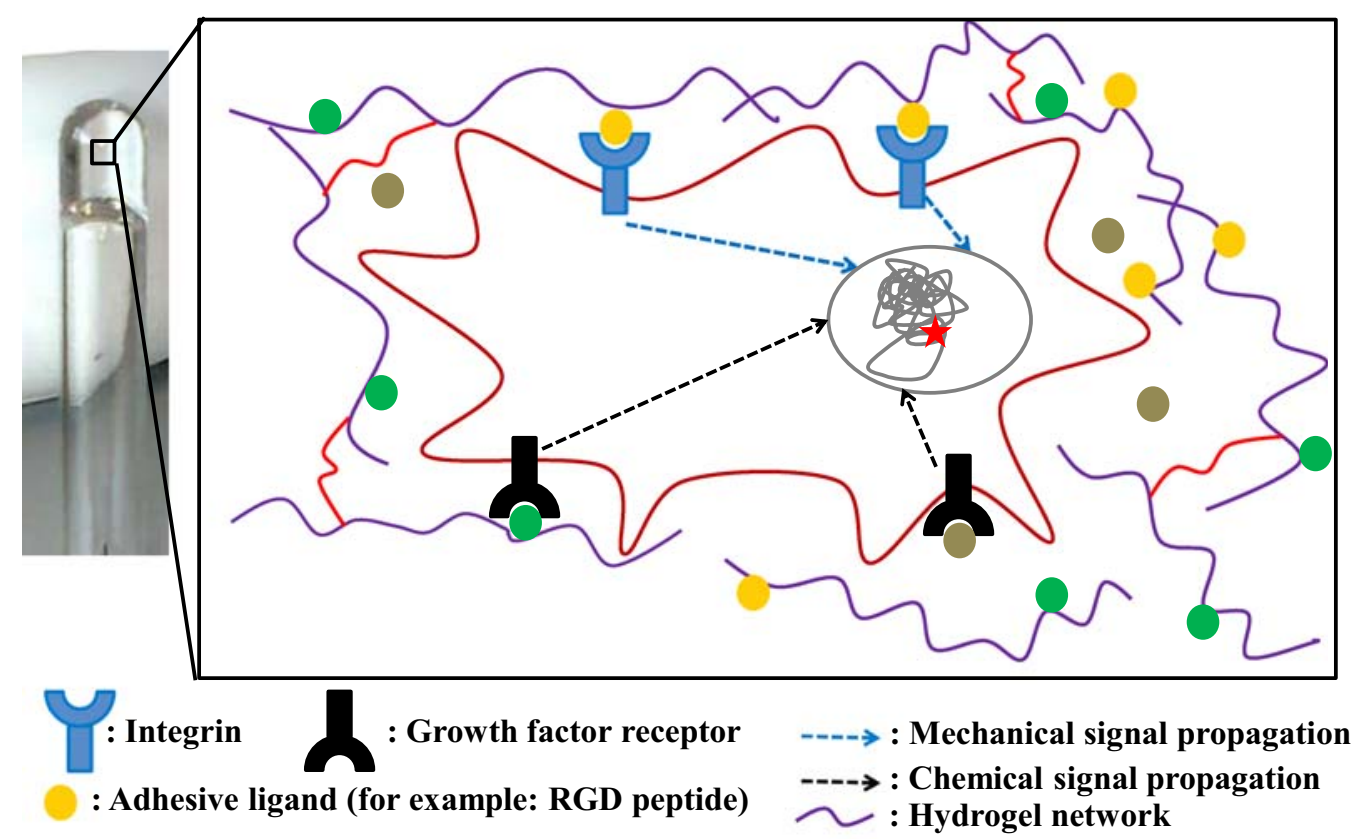

: Growth factor/Growth factor mimetic peptide (hydrogel bound)

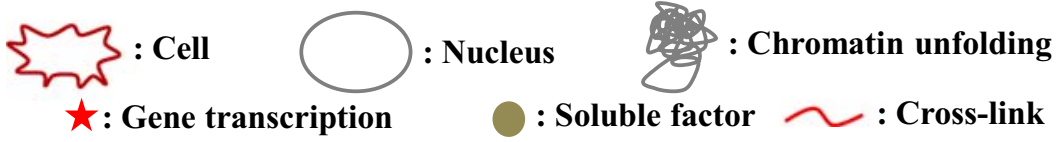

Figure 1. Schematic representation of stem cell-hydrogel interaction. Cytoskeleton-linked integrin receptor-adhesive ligand interaction provides physical cues (stiffness, topography of microenvironment, etc.). Interaction of growth factor receptor with growth factors (hydrogel bound or soluble) provides chemical cues. The mechanical and chemical signals propagate to the nucleus through different signalling pathways. Chromatin unfolding and gene expression occur in the nucleus resulting in the expression of different transcription factors and proteins. The cross-links present in the hydrogel network provide control of hydrogel mechanical properties, resulting in modulation of mechanical signals presented to the cells (different levels of control such as, temporal, spatiotemporal, etc. are possible depending on the hydrogel design principle), eventually controlling cell fate.

to spread and migrate. Natural ECM materials like fibrin and collagen contain enzymatically degradable sites. In contrast, synthetic materials, which oftentimes have average pore size of the order of $\mathrm{nm}$, much smaller than the $\mu \mathrm{m}$ sized cells, need to include crosslinkers that are susceptible to cell secreted enzymes, for example, matrix metalloproteinase (MMP) degradable cross-linkers. However, synthetic scaffolds may also incorporate cross-links that are not biodegradable, but that can be degraded on demand of the user, by an external trigger (see below).

\subsection{Functionality, adhesion and cellular migration}

Natural ECM proteins (fibrin, collagen, hyaluronic acid etc.) already contain cell-interactive sites. Design of synthetic hydrogels should take into account the inclusion of cell adhesive cues (for example, RGD). Cell adhesion is required not only to provide chemical cues between cells but also to enable the formation of biomechanical sensors that dictate the density of FA which in turn effect the intra cellular chemical signals (for example, phosphorylation of the tyrosine residue of focal adhesion kinase) that are transported to the nucleus and dictates cellular fate. Besides, covalent/non-covalent grafting of specific bioactive epitopes is necessary to elicit specific cell responses.

Importantly, cellular adhesion to the ECM is necessary for cells to generate enough traction to enable cell migration, especially in ECM materials with very small porosity $(\sim \mathrm{nm})$ compared to cellular dimension $(\sim \mu \mathrm{m})$. An optimum cell-matrix adhesive interaction has been shown to be critical for maximum speed of cell migration. Thus, migration speed shows a biphasic response to the adhesion ligand density, both in $2 \mathrm{D}^{12}$ as well as $3 \mathrm{D}$ cell culture conditions. ${ }^{13-16}$ In tissue engineering applications, increased cellular migration speed is often a desired property. ${ }^{17}$ For example, a critical step for wound repair involves invasion and partial degradation of the matrix (at the site of tissue defect) by inflammatory cells, followed by remodelling (infiltration by other cell types such as fibroblasts that 
differentiate and generate new ECM). ${ }^{18}$ Thus, an advanced synthetic construct designed to facilitate regeneration of tissue should satisfy this criterion.

\subsection{Tunable physical and chemical properties}

Different functional group grafting modes (Michael type addition, non-covalent, photopolymerization, photodegradable cross-links, etc.) should allow access to synthetic materials, which possess physical and chemical properties that can be controlled spatially and temporally.

\section{Elaboration of the design principles in synthetic/semi-synthetic hydrogel materials}

In this section, we shall discuss representative examples to demonstrate the integration of different engineering principles described in the last section. These examples are by no means exhaustive and are chosen to highlight the design principles.

\subsection{Degradability of scaffold and tunability of (chemical and physical) properties}

5.1a Hydrolytic and proteolytic degradation: Hydrolytic and proteolytic degradation are two most common strategies to render synthetic hydrogels degradable over time. Majority of examples of hydrolytically degradable hydrogels employ ester linkages as the site of hydrolysis. For instance, lactide units have been incorporated into PEG backbone to render the hydrogels degradable. ${ }^{19}$ Benoit and co-workers have developed PEG- $d$, $l$-lactide based hydrogels (as a tissue engineered periosteum) to encapsulate and localize murine MSCs around decellularized bone allografts in a rat femur model. The degradation kinetics of the hydrogel could be programmed by changing the number of lactide repeat units in the polymer backbone, thus allowing a spatiotemporal control of MSC release from the hydrogel and localization around the allograft for its subsequent healing. ${ }^{20,21}$ Elisseeff and co-workers ${ }^{22}$ have incorporated phosphoesters as degradation sites into PEG gels. These gels can be degraded both via hydrolysis as well as enzymolysis. Thus, the presence of alkaline phosphatase (ALP) accelerates the degradation. Considering that MSCs encapsulated in these gels will produce ALP as an early marker for osteogenesis (bone cell formation), the produced ALP may degrade the gel, release phosphate from the polymer, and facilitate mineralization of the gel matrix by the subsequent formation and deposition of calcium phosphate. ${ }^{22}$
For tissue engineering purpose, it is important to ensure that as the gel encapsulated cells continue depositing ECM material, and as the gel matrix also continues to degrade, the ongoing matrix degradation should not be too fast or too slow relative to the rate of cell ECM production. In this context, Anseth and co-workers reported on co-polymers of PEG and poly(vinyl)alcohol, PVA, that had a slower degradation kinetics compared to lactic acid incorporated PEG gels. ${ }^{23}$ Thus, chondrocytes entrapped in these copolymer gels produced neo-cartilage tissue in 2 weeks. The initial void spaces observed at 2 weeks were completely filled up by the generated tissue after 6 weeks. This contrasted the observation with PEG-LA gels, which degraded faster, and as a result, the neo-tissue was not able to fill up the void spaces even after 6 weeks. ${ }^{24}$ This example thus illustrates the importance of fine tuning the degradation kinetics of the engineered constructs to attain the goal of effective tissue regeneration.

Instead of a hydrolytic degradation strategy, gels can also be programmed with enzymatically degradable segments. There are broadly two strategies to achieve this purpose: (1) The gel can be integrated with a natural ECM material, such as, hyaluonic acid (HA) that is susceptible to enzymatic degradation (HA can be degraded by the action of cell secreted hyaluronidase). (2) Specific short peptide sequences degradable by cell secreted enzymes, for example, matrix metalloproteinase (MMP) cleavable sequences, can be built into the matrix design. As an example of strategy (1), methacrylated HA can be photopolymerized with/without PEGDA (bisacrylated PEG) to access gel materials with a wide range of material properties and degradation characteristics, with direct implications in tissue engineering applications. ${ }^{25,26}$ In one of the early examples of synthetic, proteolytically degradable gel materials (an example of strategy (2)), Hubbell and co-workers introduced poly(ethylene glycol) (PEG) based gels with MMP degradable cross-linkers to enable gel embedded cells to degrade the gel by cell secreted MMPs and make pathways for migration. ${ }^{27}$ Vinyl-sulfone end functionalized muti-arm PEG macromers were cross-linked by Michael-type addition with bis-cysteine peptides (containing MMP-degradable sequence) to provide gels with enzymatically degradable cross-links. ${ }^{28}$ Besides, the macromers were also reacted with mono-cysteine peptides (containing adhesive peptide sequence) to provide adhesive cues to the encapsulated cells. Human foreskin fibroblasts (HFFs) loaded in firbin clots and embedded in these hydrogels, showed radial growth into the matrix, and migrated with intact cell-cell contact. Importantly, gels with no adhesive ligands impeded cell invasion, clearly suggesting integrin-dependent cellular 
migration mode in these gels. The degree of cell invasion was shown to depend on the MMP-sensitivity of the cross-linker (control over the extent of $3 D$ matrix invasion). Thus, employing a cross-linker of higher MMP-sensitivity led to a significantly higher invasion.

\section{1b Patterning of hydrogels and tuning of proper-} ties: It is important for advanced tissue engineering scaffolds to encode instructions that allow access to user-defined control of the encapsulated cell microenvironment. For instance, spatio-temporal control of matrix degradation, as well as dynamic programming of matrix physical and chemical properties is desirable to achieve sophisticated, intelligent functional materials. Anseth and co-workers designed PEG-based gels with photodegradable cross-links to achieve spatial and temporal control of gel physical and chemical properties in the presence of encapsulated cells. ${ }^{29}$ Utilizing two units of a $o$-nitrobenzyl functionality (photodegradable), they developed a PEG-bis-acrylate cross-linker. Co-polymerization of this cross-linker with PEG-monoacrylate (PEGA) resulted in a hydrogel, the degradation of which (by breaking the photo-labile cross-links) could be externally triggered by light irradiation $(365 \mathrm{~nm})$. The important features of this study need to be highlighted: (1) The extent and rate of gel degradation could be controlled by the duration of exposure and intensity of irradiation, respectively, thus allowing temporal control of gel physical property (stiffness). (2) When the hydrogel was irradiated through a photomask (using specific wavelength of light that corresponded to high molar absorptivity of the photolabile function), the exposed areas were eroded at the surface of the hydrogel, generating distinct 2D surface features, without compromising the bulk physical properties of the hydrogel. Besides, 3D microchannels could be generated by controlled raster scan using laser scanning microscopy (LSM), which were able to direct fibrosarcoma cell migration. Thus, 2D and 3D micropatterning of hydrogel could be achieved (spatial control of gel physical properties; overall, controlled synthetic microenvironment for cell culture). (3) The study also demonstrated that by attaching a bioactive function (cell adhesive peptide RGDS) to the PEG polymer matrix through a photolabile linker (nitrobenzyl ether), the local chemical environment of the hydrogel could be modified on demand. Irradiation of this hydrogel resulted in the cleavage of the tethered RGD from the matrix and its release from the gel. Thus, encapsulation of hMSCs into these RGD-tethered PEG gels and subsequent release of RGD by photoirradiation after 10 days of culture led to (a) decreased integrin expression of hMSCs after 21 days of culture (dynamic control of cell-material chemical interaction), (b) significant increase of hMSCs chondrogenesis (directing specific stem cell functions by external trigger).

The photodegradation kinetics of $o$-nitrobenzyl $(o-\mathrm{NB})$ linkers is dependent on the structural aspects of the linker. For example, the kinetics is increased significantly if the benzylic position is secondary and if there is no $o$-aryl substituent on the ring. Exploiting this difference of reactivity of different $o$-NB linkers, sequential release of different cell populations (green fluorescent protein or, red fluorescent protein expressing hMSCs) from the PEG hydrogels has recently been demonstrated. ${ }^{30}$ Thus, synthesizing gels that incorporate $o$-NB linkers of different photodegradation kintetics and by adjusting the intensity and duration of light exposure, precise control over multistep delivery of hydrogel encapsulated cells becomes possible (encapsulation and on-demand release of therapeutic cells).

These examples indicate that incorporating multiple photocleavable units that respond to different wavelengths of light can allow access to hydrogel materials where release or patterning of multiple proteins can be performed in 'wavelength-orthogonal' fashion. ${ }^{31-33}$ Indeed, Azagarsamy and Anseth have demonstrated that covalently tethering BMP-2 and BMP-7 (bone morphogenic proteins that regulate bone regeneration) to a PEG hydrogel through $o$-NB and coumarine units that have wavelength dependent degradation kinetics (highest degradation rates at $405 \mathrm{~nm}$ and $365 \mathrm{~nm}$, respectively) can realize such responsive materials. ${ }^{34}$ Sequential release of BMP-2 and BMP-7 can be realized in presence of both the covalently grafted proteins by first irradiating the sample with $405 \mathrm{~nm}$ light (cleaves $o$ NB tethered BMP-2) and then exposing to $365 \mathrm{~nm}$ light (cleaves coumarine tethered BMP-7). This sequential release of BMP-2 and BMP-7 at user defined time points resulted in improved osteogenesis of hMSCs seeded on these gels.

Anseth and co-workers have demonstrated that by using sequential bio-orthogonal click reactions, biofunctional hydrogel materials can be generated with independent control of the physical and chemical properties of the material. ${ }^{35}$ They employed the $\mathrm{Cu}$-free Huisgen cycloaddition between a 4-arm PEG-tetraazide and a dicyclooctyne functionalized peptide (crosslinker) to obtain the hydrogel. A MMP cleavable peptide sequence was selected as the cross-linker to enable cell secreted protease mediated degradation of the gel and hence the modulation of the gel stiffness and porosity to facilitate cell spreading and migration (control of gel physical properties). The cross-linking peptide also contained a pendant allyl ester function, which 
was subsequently used to covalently tether any cysteine containing biomolecule of interest through an orthogonal thio-ene photocoupling reaction postgelation. Thus, 2D (by using photolithography) and 3D (controlling the focal point of laser confocal microscope) micropatterns could be generated by irradiation with $365 \mathrm{~nm}$ light (spatial and temporal photopatterning of hydro$\mathrm{gel}$ ) and multiple functionalities could be introduced at user defined positions in 2D and 3D (user defined control of chemical properties).

Similar to this example, Burdick and co-workers reported on a strategy of introducing multiple functionalities into the gel matrix to exercise on demand control on the modulation of the gel stiffness. They studied hMSCs differentiation in RGD modified hyaluronic acid (HA) hydrogels. ${ }^{36}$ HA was functionalized with both methacrylate and maleimide moieties. In the first step, Michael addition reaction between the maleimide and a thiol containing bifunctional MMP-degradable peptide was used to form the gel (introduction of proteolytically degradable site) along with a thiol containing RGD peptide, to provide adhesive cue to the cells. In the second step, after incubating the hMSCs for a specific time during which the cells started to secrete proteases, degrade the gel and spread, free-radical photopolymerization of the pendant methacrylate groups was carried out by exposure to UV irradiation. This produced kinetic chains that arrested proteolytic degradation of cross-links, and thus prevented further gel stiffness modulation. By executing such precise control of gel physical properties, the study was able to demonstrate matrix degradation as an independent parameter that could, independent of gel stiffness, direct stem cell differentiation.

Recently, utilizing the supramolecular interactions between alpha-cyclodextrin ( $\alpha-C D)$ and PEG, Elisseeff and co-workers designed a multifunctional hydrogel containing $\alpha$-CD threaded into PEG chains (polymer necklace). ${ }^{37}$ The polymer stiffness could be controlled simply by controlling cross-link density. The hydroxyl groups of the threaded $\alpha-C D$ could be functionalized with different bioactive functions promoting cell adhesion or specific cell fate (for example, differentiation). Thus, the concentration of the ligands can be varied independent of the stiffness of the material. This example thus introduces a modular functionalization strategy for precise control of biochemical signal presentation where chemical modification of the PEG backbone itself is not required. The rotational freedom of $\alpha-\mathrm{CD}$ on $\mathrm{PEG}$ thread further allows optimal interaction between ligand and cell surface receptors. This hydrogel has the potential to be rendered degradable by introducing a suitable cross-linker.

\subsection{Presentation of chemical signals (functionality)}

Although we have covered some aspects of ligand presentation in the previous discussion, we shall more specifically highlight some strategies of growth factor presentation in hydrogels in this section. In vivo, most of the growth factors are immobilized in ECM, because they are attached to glycosaminoglycans (GAGs) or proteins. ${ }^{38}$ Besides, it has also been demonstrated that the presentation of growth factors in matrix bound form is generally more efficient than in soluble form. For example, Griffith and co-workers were the first to report the efficacy of this mode of growth factor presentation. They demonstrated that the efficiency of mouse epidermal growth factor (EGF) to induce DNA synthesis, was not affected by its covalent tethering to a glass substrate. ${ }^{39}$ Instead of the delivery of growth factor in a large dose, slow, sustained release is often desirable. ${ }^{40}$ For example, a recent report has demonstrated a sustained delivery of bone morphogenetic protein BMP-2, as opposed to a presentation in high concentration, is more effective in repairing bone defect in a rat intramuscular ectopic model. ${ }^{41}$ Thus, synthetic/semi-synthetic hydrogel materials should target covalent grafting or sequestration of growth factors as the preferred mode of presentation to the matrix-encapsulated cells.

\section{2a Covalent immobilization of growth factors in} synthetic scaffolds: As an example of growth factor immobilization, West and co-workers covalently grafted basic fibroblast growth factor (bFGF) within a PEG scaffold. ${ }^{42}$ Hydrogels containing bGFG gradient induced smooth muscle cell (SMC) alignment and migration. Importantly, hydrogel immobilized bFGF exhibited higher efficiency than free bFGF. However, the covalent tethering of a large protein (growth factor) to the polymer scaffold is not cost-effective, and the construct may suffer from immunogenicity, reduced bioactivity ${ }^{43}$ and shorter in vivo half-life. ${ }^{44}$ Thus, an alternative strategy has evolved where short peptides mimicking the active sequence (the receptor-binding domain) of the growth factor are designed and presented in the scaffold by covalent attachment, to achieve the function of the growth factor. Zouani et al., have demonstrated that (poly(acrylamide-co-acrylic acid)) gels (stiffness: $15 \mathrm{kPa}$ ) covalently functionalized with a bone morphogenetic protein (BMP-2) mimetic peptide, can successfully induce osteogenic differentiation in hMSCs. ${ }^{45}$ The presence of grafted peptide functionality activated BMP-Smad pathway in the hMSCs, leading to nuclear translocation of Smad proteins followed by upregulation of osteogenic (bone) markers, and suppressing myogenic (muscle) differentiation pathway. 
Heilshorn and co-workers have reported covalent incorporation of a vascular endothelial growth factor (VEGF) mimetic peptide into an elastin-like polypeptide derived hydrogel. ${ }^{46}$ Human umbilical vein endothelial cells (HUVEC) encapsulated within these hydrogels not only remained viable, but also showed augmented 3D protrusions in the hydrogel, compared to pristine gels supplemented with soluble VEGF mimetic peptide at the same concentration. These examples thus showcase that, indeed, engineered short peptides incorporated into functional scaffolds can successfully mimic the functions of the growth factors in directing cell functions.

\section{2b Exploiting the ECM component-growth factor} interactions: Other than direct covalent immobilization of growth factors/mimetic peptides onto the ECM mimetic scaffold, different biomimetic strategies have also been pursued. In natural ECM, the ECM proteins play an important role to regulate growth factor signalling. Proteoglycans (consisting of a core protein, with covalently attached glycosaminoglycan, GAG chains) interact with different growth factors to direct their biological functions. For example, perlecan, a proteoglycan containing heparin sulfate (HS)/chondroitin sulfate (CS) chains as the GAG units, has both pro-angiogenic as well as anti-angiogenic function. ${ }^{47}$ To elaborate, the HS chains in perlecan upregulate fibroblast growth factor (FGF)-receptor interaction, whereas the CS chains have a downregulating effect. The different strategies aiming to localize the growth factors in hydrogel scaffold by utilizing their interaction with GAGs will be summarized by discussion of representative studies:

5.2.2a Covalent grafting of the GAGs onto the hydrogel: As an example of this approach, heparin (a glycosaminoglycan) was covalently incorporated into PEG hydrogels (co-polymerization of methacrylated heparin with di-methacrylated PEG) to allow sequestration of exogenous BMP-2 (by utilizing heparin/BMP-2 interaction) and promote osteogenic differentiation of encapsulated hMSCs. ${ }^{48}$ Localized and sustained release of growth factors was reported in heparin functionalized alginate gels grafted onto large bone defects, through direct, reversible binding of the growth factor to the hydrogel. ${ }^{49}$

Multiple GAGs have also been covalently grafted onto hydrogels so that the resulting scaffold more closely resembles the natural ECM, which does not only contain heparin, but also presents other sulfated GAGs, for example, chondroitin sulfate (CS). Thus, Cai et al., have simultaneously incorporated thiol modified CS and heparin by co-polymerizing them with PEG-bisacrylate and demonstrated that slow release of gel sequestered
FGF-2 was effective in promoting in vivo neovascularisation in a mouse model. ${ }^{50}$ These examples demonstrate that covalent immobilization of glycosaminoglycans in hydrogel scaffolds is an effective strategy to exploit the interaction of these GAGs with growth factors, resulting in their efficient sequestration in these hydrogels, and subsequent slow release.

5.2.2b Non-covalent interaction of the GAGs with the hydrogel: As an example of this strategy, Stupp and co-workers reported on a self-assembling peptide amphiphile, the positive charge of which was screened in the presence of heparin (negatively charged), leading to the formation of cylindrical nanofibers through hydrophobic collapse of the alkyl tail and $\beta$-sheet formation. The negatively charged heparin was excluded from the hydrophobic interior and localized on the surface of these nanofibers. This hydrogel, when loaded with the angiogenic growth factors VEGF and FGF-2, significantly promoted neovascularisation response in a rat cornea angiogenesis assay. ${ }^{51}$

\subsection{Topographical signals}

This section attempts to put in context the techniques that are used to generate clinically viable organized artificial matrix materials. Nanofibrillar scaffold of ECM presents topographical cues to the stem cells, influencing their actions. Organization of fibers in this scaffold has ramifications in survival and proper functioning of tissues. Tissue structural fidelity is severely compromised during disease. For example, osteogenesis imperfect, a heritable disease that is manifested in fragility in collagen-I rich tissues, is characterized by more than 70 mutations in the structural genes of type I collagen. ${ }^{52}$ Thus, it is important to consider this aspect while developing artificial tissue constructs.

There are three general strategies to prepare nanoscale ECM mimetic scaffolds for tissue engineering: (1) Selfassembly, (2) Phase separation, (3) Electrospinning.

5.3a Self-assembly: We shall discuss one representative example to illustrate how self-assembly could be used to present topographical signal to the cells. Das et al., have demonstrated that helical silica nanostructures transcribed from self-assembled amphiphiles can promote osteogenesis of hMSCs when they have a periodicity of $63 \mathrm{~nm}$ (very close to the band periodicity found in collagen I (the main collagen component of bone). ${ }^{53}$ However, when the periodicity of the nanofibers was increased to $\sim 100 \mathrm{~nm}$, osteogenesis was suppressed. This is an example where self-assembly was used as a tool to generate nanoscale periodic patterns that influenced stem cell differentiation. 
5.3b Phase separation: The basic premise of this method relies on the phase separation of polymer from its solution to form a polymer rich domain (by changing temperature, adding non-solvent, etc.). ${ }^{54}$ The polymer morphology is then fixed by low temperature quenching. Subsequent freeze-drying gets rid of the solvent, resulting in the nanoporous polymer scaffold. For example, a porous biodegradable poly (L-lactic acid) (PLLA) scaffold was prepared by this technique, through phase separation from its THF solution (by cooling down a homogeneous solution), followed by solvent leaching with water and freeze drying. Mouse cerebellum C17-2 stem cells were able to differentiate in the resultant PLLA matrix, and the matrix supported neurite outgrowth. ${ }^{55}$

5.3c Electrospinning: In this technique, an electrical force is used to eject polymer solution (or melt) through a charged needle as polymer fibers, which are then deposited on a collector. The fiber size and the mesh size of the scaffold can be tuned by controlling polymer solution concentration, potential difference between the needle and the collector, polymer flow rate, etc. Apart from self-assembly and polymer phase-separation techniques, electrospinning is the most versatile technique till date that has been utilized to generate porous, fibrous constructs of size scale similar to the native ECM, with controlled mechanical properties. For example, electrospun scaffolds of type II collagen were demonstrated to support infiltration of seeded chondrocytes, showing potential for the repair of articular cartilage. ${ }^{56}$ Kaplan and co-workers have shown that vascular cells (human aortic endothelial cells and human coronery artery smooth muscle cells) can survive and proliferate in electrospun silk fibroin scaffolds, opening up the possibility for use of such scaffolds in vascular tissue engineering. ${ }^{57}$ Burdick and co-workers created mechanical (varying cross-linker density) and adhesive (varying cell-adhesive ligand RGD density) gradient through the thickness (depth-wise gradient) of an electrospun hyaluronic acid (HA) scaffold. ${ }^{58}$ Chick aortic arch implants cultured on RGD gradient scaffolds for 7 days showed enhanced infiltration into low-high gradient scaffold (lower RGD density on the surface and higher RGD density at the bottom). These examples suggest electrospun scaffolds hold great promise for use in tissue engineering constructs.

\section{Consideration of $2 \mathrm{D}$ vs $3 \mathrm{D}$ cell culture condition}

Matrix dimesionality is increasingly being recognized as a key parameter governing cellular response. 2D culture condition forces the cells to adopt apical-basal polarity, which is not the natural polarization of all classes of cells, for example, MSCs. Unlike the 2D culture, cell migration in 3D matrix in a restricted environment depends on factors such as (1) matrix porosity (gels having porosity $\sim \mathrm{nm}$ hinder the movement of cells, which have size $\sim \mu \mathrm{m}$ ) and (2) ability of the cells to secrete proteases and degrade/remodel the matrix to make space for movement. Besides, the mechanism of cell spreading and movement may be different in 3D, when compared to $2 \mathrm{D}$. A recent report suggests that in 3D microenvironment, microtubule dynamics is important for cell migration. ${ }^{59}$ Confined environment induced actin reorganization and prevented focal adhesion maturation in human breast carcinoma cells. Blocking of actomyosin contraction using blebbistatin or blocking of $\beta 1$ integrins did not affect cellular motility. ${ }^{59}$ In contrast, in 2D condition, actomyosin contraction and focal adhesion play important roles in the process. When cultured on fibronectin coated polyacrylamide substrates of varying stiffness, fibroblasts spread to a greater area with increasing stiffness of the substrate. ${ }^{60}$ Interestingly, when encapsulated in a 3D matrix, these cells adopt spindle morphology, unaffected by the stiffness of the matrix. ${ }^{61}$ Considering that cell spreading and morphology may have implications in altering mechanical signals presented to cells, one critical question could be asked: does the matrix dimension influence eventual cell fate? Indeed, bulk matrix stiffness has been shown to direct stem cell differentiation in a morphology dependent manner in experiments conducted on 2D hydrogels. ${ }^{62}$ However, recent research in $3 \mathrm{D}$ hydrogel matrices has suggested factors such as cell traction induced matrix deformation, ${ }^{63}$ cell-mediated matrix degradation (vide supra) ${ }^{36}$ and stress-stiffening ${ }^{64}$ could provide pathways that either acting together with or independent of bulk matrix stiffness, can be important mechanical regulators of stem cell differentiation. In these situations, stem cell lineage selection is independent of cell morphology. It should thus be evident from this discussion that any consideration of biomaterial design to influence cell fate must factor in the dimensionality of the matrix.

\section{Conclusions}

In this review, we have attempted to provide a critical account of the advancement in engineered hydrogel materials as scaffolds that could mimic the extracellular environment (at nano and microscale) to direct cell functions. Although a great deal of effort has already been directed towards deciphering the cell-material interactions at the fundamental level, fabricating functional materials that could capture the spatiotemporal instructions present in native ECM both in complexity and 
functionality is still an enormous challenge. The field is highly interdisciplinary and it is envisaged that, apart from cell biologists, it will continue to witness important contributions from materials scientists and chemists in the coming years to open up exciting avenues for applications in tissue engineering and regenerative medicine.

\section{References}

1. Das R K and Zouani O F 2014 A review of the effects of the cell environment physicochemical nanoarchitecture on stem cell commitment Biomaterials $\mathbf{3 5} 5278$

2. Higuchi A, Ling Q D, Hsu S T and Umezawa A 2012 Biomimetic cell culture proteins as extracellular matrices for stem cell differentiation Chem. Rev. 1124507

3. Wei G, Su Z, Reynolds N P, Arosio P, Hamley I W, Gazit E and Mezzenga R 2017 Self-assembling peptide and protein amyloids: From structure to tailored function in nanotechnology Chem. Soc. Rev. 464661

4. Humphries J D, Wang P, Streuli C, Geiger B, Humphries $\mathrm{M} \mathrm{J}$ and Ballestrem C 2007 Vinculin controls focal adhesion formation by direct interactions with talin and actin J. Cell Biol. 1791043

5. del Rio A, Perez-Jimenez R, Liu R, Roca-Cusachs P, Fernandez J M and Sheetz M P 2009 Stretching single talin rod molecules activates vinculin binding Science 323638

6. Liu J, Wang Y, Goh W I, Goh H, Baird M A, Ruehland S, Teo S, Bate N, Critchley D R, Davidson M W and Kanchanawong P 2015 Talin determines the nanoscale architecture of focal adhesions Proc. Natl. Acad. Sci. U. S. A. $112 \mathrm{E} 4864$

7. Bell E, Ehrlich H P, Buttle D J and Nakatsuji T 1981 Living tissue formed in vitro and accepted as skin-equivalent tissue of full thickness Science 2111052

8. Yannas I V, Lee E, Orgill D P, Skrabut E M and Murphy GF 1989 Synthesis and characterization of a model extracellular matrix that induces partial regeneration of adult mammalian skin Proc. Natl. Acad. Sci. U. S. A. 86 933

9. Ma L, Gao C, Mao Z, Zhou J, Shen J, Hu Z and Han C 2003 Collagen/chitosan porous scaffolds with improved biostability for skin tissue engineering Biomaterials $\mathbf{2 4}$ 4833

10. Schroeder-Tefft J A, Bentz H and Estridge T D 1997 Collagen and heparin matrices for growth factor delivery J. Control Release $\mathbf{4 9} 291$

11. Hudalla G A and Murphy W L 2011 Biomaterials that regulate growth factor activity via bioinspired interactions Adv. Funct. Mater. 211754

12. Palecek S P, Loftus J C, Ginsberg M H, Lauffenburger D A and Horwitz A F 1997 Integrin-ligand binding properties govern cell migration speed through cell-substratum adhesiveness Nature 385537

13. Kuntz R M and Saltzman W M 1997 Neutrophil motility in extracellular matrix gels: mesh size and adhesion affect speed of migration Biophys. J. 721472

14. Schense J C and Hubbell J A 2000 Three-dimensional migration of neurites is mediated by adhesion site density and affinity J. Biol. Chem. 2756813
15. Burgess B T, Myles J L and Dickinson R B 2000 Quantitative analysis of adhesion-mediated cell migration in three-dimensional gels of RGD-grafted collagen Ann. Biomed. Eng. 28110

16. Gobin A S and West J L 2002 Cell migration through defined, synthetic extracellular matrix analogues FASEB J. 16751

17. Langer R and Vacanti J 1993 Tissue Eng. 260920

18. Clark R A F 1996 Molecular and Cellular Biology of Wound Repair (New York: Plenum)

19. Benoit D S W, Durney A R and Anseth K S 2006 Manipulations in Hydrogel Degradation Behavior Enhance Osteoblast Function and Mineralized Tissue Formation Tissue Eng. 121663

20. Hoffman M D, Xie C, Zhang X and Benoit D S W 2013 The effect of mesenchymal stem cells delivered via hydrogel-based tissue engineered periosteum on bone allograft healing Biomaterials 348887

21. Hoffman M D, Van Hove A H and Benoit D S W 2014 Degradable hydrogels for spatiotemporal control of mesenchymal stem cells localized at decellularized bone allografts Acta Biomater. 103431

22. Wang D A, Williams C G, Yang F, Cher N, Lee H and Elisseeff J H 2005 Bioresponsive phosphoester hydrogels for bone tissue engineering Tissue Eng. 11201

23. Martens P J, Bryant S J and Anseth K S 2003 Tailoring the degradation of hydrogels formed from multivinyl poly(ethlene glycol) and poly(vinyl alcohol) macromers for cartilage tissue engineering Biomacromolecules 4 283

24. Bryant S J and Anseth K S 2003 Controlling the spatial distribution of ECM components in degradable PEG hydrogels for tissue engineering cartilage J. Biomed. Mater. Res. A 6470

25. Burdick J A, Chung C, Jia X, Randolph M A and Langer, R 2005 Controlled degradation and mechanical behavior of photopolymerized hyaluronic acid networks Biomacromolecules 6386

26. Masters K S, Shah D N, Leinwand L A and Anseth K S 2005 Crosslinked hyaluronan scaffolds as a biologically active carrier for valvular interstitial cells Biomaterials 262517

27. Lutolf M P, Lauer-Fields J L, Schmoekel H G, Metters A T, Weber F E, Fields G B and Hubbell J A 2003 Synthetic matrix metalloproteinase-sensitive hydrogels for the conduction of tissue regeneration: engineering cellinvasion characteristics Proc. Natl. Acad. Sci. U. S. A. 1005413

28. Lutolf M P and Hubbell J A 2003 Synthesis and physicochemical characterization of end-linked poly (ethylene glycol) -co-peptide hydrogels formed by Michael-type addition Biomacromolecules 4713

29. Kloxin A M, Kasko A M, Salinas C N and Anseth K S 2009 Photodegradable hydrogels for dynamic tuning of physical and chemical properties Science 324 59

30. Griffin D R and Kasko A M 2012 Photodegradable macromers and hydrogels for live cell encapsulation and release J. Am. Chem. Soc. 13413103

31. Gatterdam V, Stoess T, Menge C, Heckel A and Tampé R 2012 Caged glutathione-Triggering protein interaction by light Angew. Chem. Int. Ed. $\mathbf{5 1} 3960$ 
32. Labòria N, Wieneke R and Tampé R 2013 Control of nanomolar interaction and in situ assembly of proteins in four dimensions by light Angew. Chem. Int. Ed. 52 848

33. Grunwald C, Schulze K, Reichel A, Weiss V U, Blaas D, Piehler J, Wiesmüller K H and Tampé R 2010 In situ assembly of macromolecular complexes triggered by light Proc. Natl. Acad. Sci. U. S. A. 1076146

34. Azagarsamy M A and Anseth K S 2013 Wavelengthcontrolled photocleavage for the orthogonal and sequential release of multiple proteins Angew. Chem. Int. Ed. 5213803

35. DeForest C A, Polizzotti B D and Anseth K S 2009 Sequential click reactions for synthesizing and patterning three-dimensional cell microenvironments Nat. Mater. 8659

36. Khetan S, Guvendiren M, Legant W R, Cohen D M, Chen C S and Burdick J A 2013 Degradation-mediated cellular traction directs stem cell fate in covalently crosslinked three-dimensional hydrogels Nat. Mater. 12458

37. Singh A, Zhan J, Ye Z and Elisseeff J H 2013 Modular multifunctional poly(ethylene glycol) hydrogels for stem cell differentiation Adv. Funct. Mater. 23575

38. Hynes R O 2009 The extracellular matrix: Not just pretty fibrils Science $\mathbf{3 2 6} 1216$

39. Kuhi P R and Griffth-Cima L G 1996 Tethered epidermal growth factor as a paradigm for growth factor-induced stimulation from the solid phase Nat. Med. 21022

40. Davis M E, Hsieh P C H, Takahashi T, Song Q, Zhang S, Kamm R D, Grodzinsky A J, Anversa P and Lee R T 2006 Local myocardial insulin-like growth factor 1 (IGF-1) delivery with biotinylated peptide nanofibers improves cell therapy for myocardial infarction Proc. Natl. Acad. Sci. U. S. A. 1038155

41. Bhakta G, Lim Z X H, Rai B, Lin T, Hui J H, Prestwich G D, van Wijnen A J, Nurcombe V and Cool S M 2013 The influence of collagen and hyaluronan matrices on the delivery and bioactivity of bone morphogenetic protein-2 and ectopic bone formation Acta Biomater. 9 9098

42. DeLong S A, Moon J J and West J L 2005 Covalently immobilized gradients of bFGF on hydrogel scaffolds for directed cell migration Biomaterials 263227

43. Eppler S M, Combs D L, Henry T D, Lopez J J, Ellis S G, Yi J H, Annex B H, McCluskey E R and Zioncheck T F 2002 A target-mediated model to describe the pharmacokinetics and hemodynamic effects of recombinant human vascular endothelial growth factor in humans Clin. Pharmacol. Ther. 7220

44. Ennett A B, Kaigler D and Mooney D J 2006 Temporally regulated delivery of VEGF in vitro and in vivo Clin. Exp. Rheumatol. 79176

45. Zouani O F, Kalisky J, Ibarboure E and Durrieu M C 2013 Effect of BMP-2 from matrices of different stiffnesses for the modulation of stem cell fate Biomaterials 342157

46. Cai L, Dinh C B and Heilshorn S C 2014 One-pot synthesis of elastin-like polypeptide hydrogels with grafted VEGF-mimetic peptides Biomater. Sci. 2757

47. Whitelock J M, Melrose J and Iozzo R V 2008 Diverse cell signaling events modulated by Perlecan diverse cell signaling events modulated by Perlecan Biochemistry $\mathbf{4 7}$ 11174
48. Benoit D S W, Durney A R and Anseth K S 2007 The effect of heparin-functionalized PEG hydrogels on threedimensional human mesenchymal stem cell osteogenic differentiation Biomaterials 2866

49. Kolambkar Y M, Dupont K M, Boerckel J D, Huebsch N, Mooney D J, Hutmacher D W and Guldberg R E 2011 An alginate-based hybrid system for growth factor delivery in the functional repair of large bone defects Biomaterials 3265

50. Cai S, Liu Y, Zheng Shu X and Prestwich G D 2005 Injectable glycosaminoglycan hydrogels for controlled release of human basic fibroblast growth factor Biomaterials 266054

51. Rajangam K, Behanna H A, Hui M J, Han, X, Hulvat J F, Lomasney J W and Stupp S I 2006 Heparin binding nanostructures to promote growth of blood vessels Nano Lett. 62086

52. Rauch F and Glorieux F H 2004 Osteogenesis Imperfecta Lancet 3631377

53. Das R K, Zouani O F, Labrugère C, Oda R and Durrieu M C 2013 Influence of nanohelical shape and periodicity on stem cell fate ACS Nano 73351

54. Hua F J, Kim G E, Lee J D, Son Y K and Lee D S 2002 Macroporous poly(L-lactide) scaffold 1. Preparation of a macroporous scaffold by liquid-liquid phase separation of a PLLA-dioxane-water system J. Biomed. Mater. Res. 63161

55. Yang F, Murugan R, Ramakrishna S, Wang X, Ma Y X and Wang S 2004 Fabrication of nano-structured porous PLLA scaffold intended for nerve tissue engineering Biomaterials 251891

56. Shields K J, Beckman M J, Bowlin G L and Wayne J S 2004 Mechanical properties and cellular proliferation of electrospun collagen type II Tissue Eng. 101510

57. Zhang X, Baughman C B and Kaplan D L 2008 In vitro evaluation of electrospun silk fibroin scaffolds for vascular cell growth Biomaterials 292217

58. Sundararaghavan H G and Burdick J A 2011 Gradients with depth in electrospun fibrous scaffolds for directed cell behavior Biomacromolecules 122344

59. Balzer E M, Tong Z, Paul C D, Hung W C, Stroka K M, Boggs A E, martin S S and Konstantopoulos K 2012 Physical confinement alters tumor cell adhesion and migration phenotypes FASEB J. 264045

60. Jiang G, Huang A H, Cai Y, Tanase M and Sheetz M P 2006 Rigidity sensing at the leading edge through $\alpha v \beta 3$ integrins and RPTP $\alpha$ Biophys. J. 901804

61. Cukierman E, Pankov R, Stevens D R and Yamada K M 2001 Taking cell-matrix adhesions to the third dimension Science 2941708

62. Engler A J, Sen S, Sweeney H L and Discher D E 2006 Matrix elasticity directs stem cell lineage specification Cell 126677

63. Huebsch N, Arany P R, Mao A S, Shvartsman D, Ali O A, Bencherif S A, Rivera-Feliciano J and Mooney D J 2010 Harnessing traction-mediated manipulation of the cell/matrix interface to control stem-cell fate Nat. Mater. 9518

64. Das R K, Gocheva V, Hammink R, Zouani O F and Rowan A E 2015 Stress-stiffening-mediated stem-cell commitment switch in soft responsive hydrogels Nat. Mater. 15318 